\title{
DOA Estimation of Mixed Coherent and Uncorrelated Targets Exploiting Coprime MIMO Radar
}

\author{
Si Qin, Yimin D. Zhang, and Moeness G. Amin
}

\begin{abstract}
We propose a new scheme to estimate the directions-of-arrival (DOAs) of mixed coherent and uncorrelated targets exploiting a collocated multiple-input multiple-output (MIMO) radar with transmit/receive coprime arrays. In the proposed scheme, the DOAs of the uncorrelated targets are first estimated using subspace-based methods, whereas those of the coherent targets are resolved using Bayesian compressive sensing. Compared with the previous works, the proposed approach achieves improved DOA estimation accuracy with a flexible coprime array configuration and may resolve more targets than the number of coarray elements. Theoretical analysis and simulation results validate the effectiveness of the proposed technique.
\end{abstract}

\section{Index Terms}

Coprime array, sum coarray, direction-of-arrival estimation, coherent targets, Bayesian compressive sensing.

\section{INTRODUCTION}

By determining the spatial spectra of the impinging targets, direction-of-arrival (DOA) estimation on multi-antenna receivers plays an important role in broad applications of wireless communications, radar, and biomedical systems. Among the many existing DOA estimation methods, subspace-based approaches, such as MUSIC [2] and ESPRIT [3], are commonly used

Part of the results was presented in the IEEE Benjamin Franklin Symposium on Microwave and Antenna Sub-systems, Philadelphia, PA, September 2014 [1].

S. Qin and M. G. Amin are with the Center for Advanced Communications, Villanova University, Villanova, PA 19085, USA.

Y. D. Zhang is with the Department of Electrical and Computer Engineering, Temple University, Philadelphia, PA, 19122, USA. (e-mail: ydzhang@temple.edu) 
to resolve uncorrelated targets because of their low complexity and superior performance. However, a major drawback with subspace-based DOA estimation methods lies in their incapability of reliably estimating DOAs for coherent targets. Coherent signal arrivals may frequently be encountered in practice due to, for example, multipath propagation caused by various reflective surfaces or closely located targets. Multiple smart jamming signals can also be designed to use coherent waveforms. The problem of DOA estimation becomes even more challenging when a mixture of coherent and uncorrelated targets is present.

In order to decorrelate the coherent targets, a number of effective approaches have been proposed, most notably is spatial smoothing technique $[4,5]$. This technique is applied prior to performing DOA estimation and divides the entire array into multiple overlapping subarrays. Averaging the output subarray covariance matrices is then performed to construct the spatially smoothed covariance matrix. Despite its effectiveness, major drawbacks of the spatial smoothing method lie in its reduction of the array aperture and the requirement of uniformly spaced sensor positions. Significant efforts have been made to develop techniques for the detection of a large number targets impinging on the array. In particular, several methods have been proposed to estimate DOAs for a mixture of both uncorrelated and coherent targets in the field of view. In [6], a uniform linear array (ULA) is considered, and the DOAs of the uncorrelated targets are first estimated by directly applying the MUSIC algorithm. The uncorrelated targets are then removed from the received data by exploiting the symmetry property of the ULA. A Toeplitz matrix is subsequently constructed to estimate the DOAs of the remaining coherent targets. In so doing, this technique enables the DOA estimation of more sources than sensors. Eliminations of uncorrelated targets can also be achieved using oblique projection as proposed [7, 8].

The aforementioned schemes employ passive or receive-only ULA for DOA estimation. Recently, multiple-input multiple-output (MIMO) has emerged to be the preferred system configuration due to, primarily, its associated long virtual array, which arises under the sum coarray equivalence in active sensing of targets [9-11]. To achieve a high number of degrees of freedom (DOFs) and improved resolution, it is desirable to have sparse transmit and receive arrays. As such, the resulting sum coarray may not have a ULA structure. This disallows spatial smoothing and, as such, renders subspace-based approaches, including the MUSIC algorithm, inapplicable.

In this paper, we consider DOA estimation exploiting a coprime MIMO radar in the presence of both uncorrelated and coherent targets. The coprime array configuration is originally proposed in [12] for passive signal reception and consists of two ULAs, where the number of antennas and 
the interelement spacing are constrained by a coprime relationship. Under the difference coarray equivalence, a coprime array of $\mathcal{O}(M+N)$ total physical sensors achieves $\mathcal{O}(M N)$ DOFs. The coprime array concept is generalized in [13] through the compression of the interelement spacing of one constituting subarray, allowing flexible array configuration design with a higher number of DOFs. It was shown that the nested array structure [14] is a special case of the generalized coprime array structure.

We employ a pair of coprime ULAs within the MIMO framework in which the generalized coprime array is examined in a sum coarray context rather than a difference coarray. That is, one ULA is used for signal emission, whereas the other acts as a receiver. We use this scheme to provide new insights into the generalized coprime array for effective DOA estimations. Given a specific number of physical sensors, the resulting coprime MIMO array structure achieves a large virtual array aperture and offers a high flexibility in trading off spatial resolution and false spectrum peaks. In this paper, in order to overcome the false peak problem, which is more likely to occur in non-uniform sum coarrays, reliable DOA estimation of coherent targets is sought out based on compressive sensing (CS) [15] techniques. These techniques do not require the Toelitz matrix structure, thereby facilitating the use of general symmetric sum coarray structures, instead of the much more strict ULA requirement of the existing methods.

A number of effective CS algorithm are available (e.g., [16-18]). As a preferred approach, we consider the problem in the Bayesian compressive sensing (BCS) or sparse Bayesian learning [19-23] as they achieve superior performance and are insensitive to the coherence of dictionary entries. To handle the complex-valued observations and entries in the underlying problem, the complex multitask Bayesian compressive sensing (CMT-BCS) [24] is used in this paper. The CMT-BCS achieves improved sparse signal reconstruction because it utilizes the group sparsity of the real and imaginary components of a complex variable. The superiority of the CMT-BCS has been successfully demonstrated in various applications, including DOA estimation, radar imaging, target localization, and time-frequency analysis [25-28].

The contribution of this paper is threefold: (1) We utilize a coprime MIMO structure as an effective platform to achieve high-resolution DOA estimation where the number of resolvable targets is significantly higher than conventional array and MIMO structures; (2) We examine the performance of different generalized coprime array structures in the context of sum coarray, and address the different features of the sum coarrays as compared to the situation when the generalized coprime array is used in the passive sense to yield difference coarrays; (3) Based on 
the symmetry of the sum coarray, we extract coherent target signals, whose DOA estimation is reformulated as a group sparse reconstruction problem. Because of its capability of utilizing all the DOFs of the coarray provided by both contiguous and discontinuous coarray sensor positions, the proposed approach achieves a higher number of resolvable coherent targets and improved performance as compared to subspace-based counterparts.

In summary, the proposed technique solves DOA estimation of mixed coherent and uncorrelated targets, and offers a much higher number of DOFs as compared to existing methods. The effectiveness and the robustness of the proposed methods are evidently demonstrated using extensive simulation results.

The remainder of the paper is organized as follows. In Section II, we describe the signal model of DOA estimation in the presence of mixed coherent and uncorrelated targets exploiting a generalized coprime MIMO radar. Then, the proposed method to solve this problem is presented in Section III, and an analytical expression of the number of DOFs is provided. Simulation results are provided in Section IV to demonstrate the effectiveness of the proposed method. Section V concludes this paper.

Notations: We use lower-case (upper-case) bold characters to denote vectors (matrices). In particular, $\mathbf{I}_{N}$ denotes the $N \times N$ identity matrix. (.)* implies complex conjugation, whereas $(.)^{T}$ and $(.)^{H}$, respectively, denote transpose and conjugate transpose. $\|\cdot\|_{2}$ represents the Euclidean $\left(l_{2}\right)$ norm, and $\mathrm{E}(\cdot)$ stands for the statistical expectation operator. $\delta_{(i, k)}$ is a delta function which takes value of 1 if $i=k$ and 0 otherwise. $\otimes$ denotes the Kronecker product. $P_{r}(\cdot)$ denotes the probability density function (pdf), and $\mathcal{N}(x \mid a, b)$ denotes that random variable $x$ follows a Gaussian distribution with mean $a$ and variance $b \cdot \operatorname{Re}(x)$ and $\operatorname{Im}(x)$ denote the real and imaginary parts of complex element $x$, respectively. $\operatorname{Tr}(\mathbf{A})$ and and $|\mathbf{A}|$ respectively returns the trace and determinant of matrix A. In addition, $\lfloor\cdot\rfloor$ denotes the floor function that returns the largest integer not exceeding the argument. $\operatorname{spark}(\mathbf{A})$ denotes the spark of $\mathbf{A}$, returning the smallest integer $r$ such that every column of $\mathbf{A}$ are linearly independent.

\section{Signal MODEL}

We consider an MIMO radar with $N$ transmit and $M$ receive antennas. As illustrated in Fig. 1, the transmit and receive arrays are collocated and they adopt the generalized coprime array configuration, where the two integers $M$ and $N$ are chosen to be coprime, i.e., their greatest common divisor is one. The unit interelement spacing $d$ is set to $\lambda / 2$, where $\lambda$ denotes the wavelength. 
Compared with the original coprime array configuration, the generalized configuration introduces an integer compression factor $p$ to compress the interelement spacing of one constituting array. Without loss of generality, we consider that the interelement spacing compression occurs in the transmit array. Alternatively, depending on the particular applications and geometric concerns, the spacing compression can be placed in the receive array. Let

$$
M=p \breve{M},
$$

for some integers $p$ and $\breve{M}$, where $2 \leq p \leq M$. Note that $\breve{M}$ and $N$ are also coprime since $M$ and $N$ do not have common factors other than unity. As such, the $M$-element receive array uses an interelement spacing of $N d$, whereas the interelement spacing of the $N$-element transmit array is compressed to $\breve{M} d=M d / p$.

In this paper, we focus on the DOA estimation problem using coprime MIMO radar, and no clutter is considered. Assume that the $N$ transmit antennas emit $N$ orthogonal waveforms with unit energy. These signals are reflected at $Q$ far-field targets, which are unresolvable through range discrimination. Their reflectivities, described as $s_{q}(t), t=1, \ldots, T$, for $q=1, \ldots, Q$, are assumed to be mixed coherent and uncorrelated targets, which are common in multipath propagation environments due to the reflection in practical situation. The angles of the $Q$ target returns are respectively denoted as $\Theta=\left[\theta_{1}, \ldots, \theta_{Q}\right]^{T}$. Then, the matched filter at the output of the $m$ th receive antenna, corresponding to the $n$th transmit waveform, generates

$$
x_{m, n}(t)=\sum_{q=1}^{Q} a_{t_{n}}\left(\theta_{q}\right) a_{r_{m}}\left(\theta_{q}\right) s_{q}(t)+n_{m, n}(t),
$$

where

$$
\begin{aligned}
a_{t_{n}}\left(\theta_{q}\right) & =e^{\frac{-j 2 \pi \dddot{M} n d}{\lambda} \sin \left(\theta_{q}\right)}, \\
a_{r_{m}}\left(\theta_{q}\right) & =e^{\frac{-j 2 \pi N m d}{\lambda} \sin \left(\theta_{q}\right)},
\end{aligned}
$$

and $n_{m, n}(t)$ is assumed to be an independent and identically distributed (i.i.d.) random variable following the complex Gaussian distribution $\mathcal{C N}\left(0, \sigma_{n}^{2}\right)$, where $0 \leq m \leq M-1$ and $0 \leq n \leq$ $N-1$. 
Stacking the output signals into a vector received at sum coarray

$$
\begin{aligned}
\mathbf{x}(t)= & {\left[x_{1,1}(t), x_{2,1}(t), \ldots, x_{M, 1}(t), x_{1,2}(t), x_{2,2}(t), \ldots,\right.} \\
& \left.x_{M, 2}(t), \ldots, x_{1, N}(t), x_{2, N}(t), \ldots, x_{M, N}(t)\right]^{T} \\
= & \sum_{q=1}^{Q} \mathbf{a}_{t}\left(\theta_{q}\right) \otimes \mathbf{a}_{r}\left(\theta_{q}\right) s_{q}(t)+\mathbf{n}(t)
\end{aligned}
$$

where

$$
\begin{aligned}
& \mathbf{a}_{t}\left(\theta_{q}\right)=\left[a_{t_{1}}\left(\theta_{q}\right), a_{t_{2}}\left(\theta_{q}\right), \ldots, a_{t_{N}}\left(\theta_{q}\right)\right]^{T}, \\
& \mathbf{a}_{r}\left(\theta_{q}\right)=\left[a_{r_{1}}\left(\theta_{q}\right), a_{r_{2}}\left(\theta_{q}\right), \ldots, a_{r_{M}}\left(\theta_{q}\right)\right]^{T}
\end{aligned}
$$

are the transmit and receive steering vectors corresponding to $\theta_{q}$, respectively, and $\mathbf{n}(t)=$ $\left[n_{1,1}(t), n_{2,1}(t), \ldots, n_{M, N}(t)\right]^{T}$ is the noise vector. As a result, a sum coarray is formed at the output of the receiver matched filters, and their positions are expressed as

$$
\mathbb{P}_{\mathrm{s}}=\{(\breve{M} n+N m) d \mid 0 \leq n \leq N-1,0 \leq m \leq M-1\}
$$

Because $M$ and $N$ are coprime, there are $M N$ unique virtual sensor positions and, therefore, the number of the available DOFs is always $M N-1$. This is different from the difference coarray case, where the number of DOFs may become smaller than $M N-1$ because of overlapped virtual sensor positions. On the other hand, the aperture of the coarray is $\breve{M}(N-1)+N(M-1)$, which, for a specific pair $M$ and $N$, increases with $\breve{M}$. In other words, a smaller value of compression factor $p$ results in a larger aperture. In addition, it could be inferred that the sum coarray is symmetric because both transmit and receive arrays are uniform linear and, thereby, symmetric.

An example is illustrated in Fig. 2, where $M=4, N=3$ and different values of the compression factor $p$ are considered. It is noted that all the resulting sum coarrays have $M N=12$ distinct elements. In addition, the sum coarrays are symmetric, but generally have missing positions (indicated by $\times$ in the figure) except for the nested case $(p=4)$. As $p$ decreases, the aperture of the sum coarray increases, and the number of missing positions increases as well. In this case, when dealing with coherent targets, subspace-based methods suffer from reduced DOFs because only contiguous coarray sensor positions can be utilized. On the other hand, the proposed CS-based approach can utilize all the DOFs provided by both contiguous and discontinuous coarray sensor positions. 


\section{PROPOSED APPROACH}

\section{A. Formulation of the Problem}

The coherent reflection signal arrivals are grouped into $V$ groups, $s_{c}^{v}(t), v=1, \ldots, V$, where the signal arrivals within each group are coherent to each other but are uncorrelated to signals in other groups. In the $v$ th group, there are $L_{v}$ coherent arrivals and the DOA corresponding to the $l$ th arrival is denoted as $\theta_{c}^{v_{l}}, l=1, \ldots, L_{v}$. The total number of coherent arrivals is $L=\Sigma_{v=1}^{V} L_{v}$

and the remaining $D=Q-L$ targets, $s_{u}^{d}(t), d=1, \ldots, D$, are uncorrelated to each other and to the coherent ones. For the convenience of presentation, we assume $M N$ to be odd and express it as $M N=2 K+1$. This occurs when both $M$ and $N$ are odd integers. However, $M N$ can take an even value and the problem can be similarly formulated.

Let the index of the central element of the array to be 0. The signal vector in Eqn. (3) can be reformulated as

$$
\begin{aligned}
\mathbf{x}(t) & =\left[x_{-K}(t), \ldots, x_{0}(t), \ldots, x_{K}(t)\right]^{T} \\
& =\sum_{v=1}^{V} \sum_{l=1}^{L_{v}} \mathbf{a}\left(\theta_{c}^{v_{l}}\right) \rho_{v_{l}} s_{c}^{v}(t)+\sum_{d=1}^{D} \mathbf{a}\left(\theta_{u}^{d}\right) s_{u}^{d}(t)+\mathbf{n}(t) \\
& =\mathbf{A}_{C} \mathbf{s}_{C}(t)+\mathbf{A}_{U} \mathbf{s}_{U}(t)+\mathbf{n}(t),
\end{aligned}
$$

where $\mathbf{a}(\theta)=\mathbf{a}_{t}(\theta) \otimes \mathbf{a}_{r}(\theta)$ is the steering vector of the sum coarray corresponding to $\theta$, and $\rho_{v l}$ is the complex coefficient of the $l$ th arrival in the $v$ th group for $l=1, \ldots, L_{v}$ with $v=1, \ldots, V$. In addition, the manifolds of the coherent and uncorrelated targets are grouped as $\mathbf{A}_{C}=\left[\mathbf{A}_{1} \boldsymbol{\rho}_{1}, \ldots, \mathbf{A}_{V} \boldsymbol{\rho}_{V}\right], \mathbf{A}_{U}=\left[\mathbf{a}\left(\theta_{u}^{1}\right), \ldots, \mathbf{a}\left(\theta_{u}^{D}\right)\right]$, where $\boldsymbol{\rho}_{v}=\left[\rho_{v_{1}}, \ldots, \rho_{v_{L_{v}}}\right]^{T}, \mathbf{A}_{v}=$ $\left[\mathbf{a}\left(\theta_{c}^{v_{1}}\right), \ldots, \mathbf{a}\left(\theta_{c}^{v_{L_{v}}}\right)\right]$, and the corresponding signal vectors are denoted as $\mathbf{s}_{C}=\left[s_{c}^{1}(t), \ldots, s_{c}^{V}(t)\right]^{T}$, $\mathbf{s}_{U}=\left[s_{u}^{1}(t), \ldots, s_{u}^{D}(t)\right]^{T}$.

As a consequence, the covariance matrix of the coarray output can be expressed as

$$
\mathbf{R}_{\mathbf{x x}}=\mathrm{E}\left\{\mathbf{x}(t) \mathbf{x}^{H}(t)\right\}=\mathbf{A}_{C} \mathbf{R}_{C} \mathbf{A}_{C}^{H}+\mathbf{A}_{U} \mathbf{R}_{U} \mathbf{A}_{U}^{H}+\sigma_{n}^{2} \mathbf{I}_{2 K+1},
$$

where $\mathbf{R}_{C}$ and $\mathbf{R}_{U}$ are the covariance matrix of $\mathbf{s}_{C}(t)$ and $\mathbf{s}_{U}(t)$, respectively. Note that $\mathbf{R}_{U}$ is a diagonal matrix, whereas $\mathbf{R}_{C}$ is block diagonal with $V$ blocks, whose rank is $V$.

\section{B. DOA Estimation}

DOA estimation is separately performed for coherent and uncorrelated targets in two steps. The first step is similar to $[6-8,29]$, in which the uncorrelated targets are estimated by directly 
applying a subspace based method, e.g., the MUSIC algorithm. After eliminating the contribution of uncorrelated targets by exploiting the property of a symmetric array that the received signal vector is conjugate symmetric, the DOAs of the coherent targets are estimated using a CS technique. The CS-based approach enables the proposed use of coprime arrays with a nonuniform sum coarray as it only requires a symmetric, instead of uniform linear, sum coarray structure.

All arrivals corresponding to the same coherent targets yield a rank-1 component. Therefore, the covariance matrix $\mathbf{R}_{\mathbf{x x}}$ can be written as

$$
\mathbf{R}_{\mathbf{x x}}=\mathbf{U}_{s} \Lambda_{s} \mathbf{U}_{s}^{H}+\mathbf{U}_{n} \boldsymbol{\Lambda}_{n} \mathbf{U}_{n}^{H}
$$

where $\mathbf{U}_{s}$ represents the signal subspace consisting of the $D+V$ dominant eigenvectors of $\mathbf{R}_{\mathbf{x x}}$ and $\mathbf{U}_{n}$ represents the noise subspace with the remaining $2 K+1-D-V$ eigenvectors. $\boldsymbol{\Lambda}_{s}$ and $\Lambda_{n}$ are diagonal matrices containing the corresponding eigenvalues. The uncorrelated targets can be estimated by exploiting the MUSIC algorithm directly. Because the combined array steering vector from multiple coherent arrivals, denoted as $\mathbf{a}_{v}=\sum_{l=1}^{L_{v}} \mathbf{a}\left(\theta_{c}^{v_{l}}\right) \rho_{v l}, v=1, \ldots, V$, does not represent a valid array manifold, the coherent targets generally do not demonstrate a strong presence in the estimated MUSIC spectra. In other words, the effect of coherent targets in each group cannot be equivalently represented as a virtual signal that would be confused with the uncorrelated targets.

Next, uncorrelated targets are eliminated by utilizing the symmetric configuration of the sum coarray. The $(i, k)$ th element of matrix $\mathbf{R}_{\mathbf{x x}}$ in (7) is expressed as

$$
r_{i, k}=\sum_{v=1}^{V} \sum_{l=1}^{L_{v}} c_{i}^{v_{l}} e^{j \pi k \sin \left(\theta_{c}^{v_{l}}\right)}+\sum_{d=1}^{D} u_{i}^{d} e^{j \pi k \sin \left(\theta_{u}^{d}\right)}+\sigma_{n}^{2} \delta_{(i, k)},
$$

for $i, k=-K, \ldots, K$, where $c_{i}^{v_{l}}=\sigma_{v}^{2} \rho_{v l}^{*} \sum_{p=1}^{L_{v}} \rho_{v p} e^{-j \pi i \sin \left(\theta_{c}^{v_{p}}\right)}$ and $u_{i}^{d}=\sigma_{d}^{2} e^{-j \pi i \sin \left(\theta_{u}^{d}\right)}$. It is noted that the uncorrelated components in $r_{i, k}$ are conjugate symmetric with respect to $i$ and $k$, while the coherent components are not because of the complex coefficient $\rho$.

Since the sum coarray configuration of the coprime array is symmetric, we can define

$$
z_{i, k}=r_{i, k}-r_{-i,-k}^{*}=\sum_{v=1}^{V} \sum_{l=1}^{L_{v}} q_{i}^{v_{l}} e^{j \pi k \sin \left(\theta_{c}^{v_{l}}\right)}+\epsilon_{i, k},
$$

where $q_{i}^{v_{l}}=\sigma_{v}^{2} \sum_{p=1}^{L_{v}}\left(\rho_{v l}^{*} \rho_{v p}-\rho_{v l} \rho_{v p}^{*}\right) e^{-j \pi i \sin \left(\theta_{c}^{v_{p}}\right)}$. In addition, an error term $\epsilon_{i, k}$ is included in Eqn.(9) to account for the discrepancies between the statistical expectation and the sample average in computing the covariance matrix. The discrepancies are modelled as i.i.d. complex 
Gaussian as a result of a sufficiently large number of samples employed in the averaging. Note that only the coherent part remains in $z_{i, k}$, as the uncorrelated components are completely eliminated.

Stacking $z_{i, k}$ for all $k$ results in $\mathbf{z}_{i}$, denoted as

$$
\mathbf{z}_{i}=\mathbf{A q}_{i}+\boldsymbol{\epsilon}_{i}
$$

where the $(2 K+1) \times L$ manifold matrix is given as $\mathbf{A}=\left[\mathbf{a}\left(\theta_{c}^{1_{1}}\right), \ldots, \mathbf{a}\left(\theta_{c}^{1_{L_{1}}}\right), \ldots, \mathbf{a}\left(\theta_{c}^{V_{1}}\right), \ldots, \mathbf{a}\left(\theta_{c}^{V_{L_{V}}}\right)\right]$ and the corresponding $L \times 1$ signal vector $\mathbf{q}_{i}=\left[q_{i}^{1_{1}}, \ldots, q_{i}^{1_{L_{1}}}, \ldots \ldots, q_{i}^{V_{1}}, \ldots, q_{i}^{V_{L_{V}}}\right]^{T}$, for $-K \leq i \leq K$. In addition, $\boldsymbol{\epsilon}_{i}$ is the error vector. It is noted that the $(2 K+1) \times 1$ vector $\mathbf{z}_{i}$ can be sparsely represented in the spatial domain over the entire angular grids as

$$
\mathbf{z}_{i}=\tilde{\mathbf{A}} \tilde{\mathbf{q}}_{i}+\boldsymbol{\epsilon}_{i}, \quad-K \leq i \leq K
$$

where $\tilde{\mathbf{A}}$ is defined as a collection of steering vectors $\mathbf{a}\left(\theta_{g}\right)$ over the entire possible grids $\theta_{g}$ for $g=1, \ldots, G$, with $G \gg L$. It is important to note that the angle positions of the coherent targets $\theta_{c}^{v_{l}}, l=1, \ldots, L_{v}, v=1, \ldots, V$, are indicated by the non-zero entries in vector $\tilde{\mathbf{q}}_{i}$, whose values describe the corresponding coefficients associated with target reflections. Generally, the non-zero entries take different values with respect to the different rows of matrix $\mathbf{R}_{\mathbf{x x}}$ (i.e., different values of $i$ ) but share the same positions because they correspond to the DOAs of the same $L$ targets. Therefore, $\tilde{\mathbf{q}}_{i}$ exhibits a group sparsity and, as such, the DOA estimation of coherent signal problem can be solved in the context of group sparse reconstruction.

To solve the complex-valued group sparse reconstruction problem in Eqn. (11), a number of effective algorithms are available under the convex optimization [30,31] and Bayesian sparse learning [21, 24] frameworks. In this paper, we exploit the CMT-BCS algorithm [24] due to its superior performance and robustness to dictionary coherence.

\section{CMT-BCS algorithm}

In this subsection, we briefly review the CMT-BCS approach based on [24] for completeness. Because it is difficult to provide a comprehensive description of the CMT-BCS within the limited space, interested readers are referred to reference [24] for CMT-BCS and references [19-23] for the general BCS framework.

Assume that the entries in the sparse vectors $\tilde{\mathbf{q}}_{i}$ are drawn from the product of the following zero-mean Gaussian distributions:

$$
\tilde{\mathbf{q}}_{i}^{g} \sim \mathcal{N}\left(\tilde{\mathbf{q}}_{i}^{g} \mid \mathbf{0}, \alpha_{g} \mathbf{I}_{2}\right), \quad g \in[1, \ldots, G]
$$


where $\tilde{\mathbf{q}}_{i}^{g}$ is a vector consisting of the real part coefficient, $\tilde{\mathbf{q}}_{i}^{g_{R}}$, and the imagery part coefficient, $\tilde{\mathbf{q}}_{i}^{g_{I}}$, with respect to the $g$ th grid in $\tilde{\mathbf{q}}_{i}$. In addition, $\boldsymbol{\alpha}=\left[\alpha_{1}, \ldots, \alpha_{G}\right]^{T}$ is a vector that contains variance of $\tilde{\mathbf{q}}_{i}^{g} g=1, \ldots, G$. Note that $\boldsymbol{\alpha}$ is shared by all groups to enforce the group sparsity. It is easy to confirm that $\tilde{\mathbf{q}}_{i}^{g}$ trends to be zero when $\alpha_{g}$ is set to zero [20].

To encourage the sparsity of $\tilde{\mathbf{q}}_{i}$, a Gamma prior is placed on $\alpha_{g}^{-1}$, which is conjugate to the Gaussian distribution, i.e.,

$$
\alpha_{g}^{-1} \sim \operatorname{Gamma}\left(\alpha_{g}^{-1} \mid a, b\right), \quad g \in[1, \ldots, G]
$$

where $\operatorname{Gamma}\left(x^{-1} \mid a, b\right)=\Gamma(a)^{-1} b^{a} x^{-(a-1)} e^{-\frac{b}{x}}$, with $\Gamma(\cdot)$ denoting the Gamma function, and $a$ and $b$ are hyper-parameters.

As the covariance matrix is estimated from the received data samples, a Gaussian prior $\mathcal{N}\left(\mathbf{0}, \beta_{0} \mathbf{I}_{2}\right)$ is also placed on the error vector $\boldsymbol{\epsilon}_{i}$. Similarly, the Gamma prior is placed on $\beta_{0}^{-1}$ with hyper-parameters $c$ and $d$.

The CMT-BCS algorithm carries out Bayesian inference by the Gibbs samplers [32]. Once the hyper-parameters $\alpha$ and $\beta_{0}$ are estimated by maximizing the marginal likelihood, the joint posterior density function of $\tilde{\mathbf{q}}_{i}^{R I}$ can be obtained analytically using Bayes' rule.

Define $\tilde{\mathbf{q}}_{i}^{R I}=\left[\left(\tilde{\mathbf{q}}_{i}^{R}\right)^{T},\left(\tilde{\mathbf{q}}_{i}^{I}\right)^{T}\right]^{T}$, with $\tilde{\mathbf{q}}_{i}^{R}=\left[\tilde{q}_{i}^{1 R}, \ldots, \tilde{q}_{i}^{G R}\right]^{T}$ and $\tilde{\mathbf{q}}_{i}^{I}=\left[\tilde{q}_{i}^{1 I}, \ldots, \tilde{q}_{i}^{G I}\right]^{T}$, the posterior for $\tilde{\mathbf{q}}_{i}^{R I}$ can be expressed analytically as a multivariate Gaussian distribution with mean $\boldsymbol{\mu}_{i}$ and covariance $\boldsymbol{\Sigma}_{i}$, i.e.,

$$
\operatorname{Pr}\left(\tilde{\mathbf{q}}_{i}^{R I} \mid \mathbf{z}_{i}, \tilde{\mathbf{A}}_{i}, \boldsymbol{\alpha}, \beta_{0}\right)=\mathcal{N}\left(\tilde{\mathbf{q}}_{i}^{R I} \mid \boldsymbol{\mu}_{i}, \boldsymbol{\Sigma}_{i}\right),
$$

where

$$
\begin{aligned}
\boldsymbol{\mu}_{i} & =\beta_{0}^{-1} \boldsymbol{\Sigma}_{i} \boldsymbol{\Psi}_{i}^{T} \mathbf{z}_{i}^{R I}, \\
\boldsymbol{\Sigma}_{i} & =\left[\beta_{0}^{-1} \boldsymbol{\Psi}_{i}^{T} \boldsymbol{\Psi}_{i}+\mathbf{F}^{-1}\right]^{-1},
\end{aligned}
$$

with

$$
\begin{aligned}
\mathbf{z}_{i}^{R I} & =\left[\operatorname{Re}\left(\mathbf{z}_{i}^{T}\right), \operatorname{Im}\left(\mathbf{z}_{i}^{T}\right)\right]^{T}, \\
\boldsymbol{\Psi}_{i} & =\left[\begin{array}{cc}
\operatorname{Re}\left(\tilde{\mathbf{A}}_{i}\right) & -\operatorname{Im}\left(\tilde{\mathbf{A}}_{i}\right) \\
\operatorname{Im}\left(\tilde{\mathbf{A}}_{i}\right) & \operatorname{Re}\left(\tilde{\mathbf{A}}_{i}\right)
\end{array}\right], \\
\mathbf{F} & =\operatorname{diag}\left(\alpha_{1}, \ldots, \alpha_{G}, \alpha_{1}, \ldots, \alpha_{G}\right) .
\end{aligned}
$$


Note that the mean and variance of each scattering coefficients can be derived using Eqns. (14) and (15) when $\boldsymbol{\alpha}$ and $\beta_{0}$ are given. On the other hand, the values of $\boldsymbol{\alpha}$ and $\beta_{0}$ are determined by maximizing the logarithm of marginal likelihood, i.e.,

$$
\left\{\boldsymbol{\alpha}, \beta_{0}\right\}=\arg \max _{\boldsymbol{\alpha}, \beta_{0}} \mathcal{L}\left(\boldsymbol{\alpha}, \beta_{0}\right)
$$

where

$$
\mathcal{L}\left(\boldsymbol{\alpha}, \beta_{0}\right)=\sum_{i=-K}^{K} \log \operatorname{Pr}\left(\tilde{\mathbf{q}}_{i}^{R I} \mid \boldsymbol{\alpha}, \beta_{0}\right)=\mathrm{const}-\frac{1}{2} \sum_{i=-K}^{K} \log \left|\mathbf{C}_{i}\right|+\left(\mathbf{z}_{i}^{R I}\right)^{T} \mathbf{C}_{i}^{-1} \mathbf{z}_{i}^{R I}
$$

and $\mathbf{C}_{i}=\beta_{0} \mathbf{I}+\boldsymbol{\Psi}_{i} \mathbf{F} \boldsymbol{\Psi}_{i}^{T}$. A type-II ML approximation [19] employs the point estimates for $\boldsymbol{\alpha}$ and $\beta_{0}$ to maximize Eqn. (20), which can be implemented via the expectation maximization (EM) algorithm to yield

$$
\begin{aligned}
& \alpha_{g}^{\text {(new) }}=\frac{1}{2 K+1} \sum_{i=-K}^{K}\left(\mu_{i, g}^{2}+\mu_{i, g+G}^{2}+\Sigma_{i, g g}+\Sigma_{i,(g+G)(g+G)}\right), \quad g \in[1, \ldots, G], \\
& \beta_{0}^{\text {(new) }}=\frac{1}{2 G(2 K+1)} \sum_{i=-K}^{K}\left(\operatorname{Tr}\left[\boldsymbol{\Sigma}_{i} \boldsymbol{\Psi}_{i} \boldsymbol{\Psi}_{i}^{T}\right]+\left\|\mathbf{z}_{i}^{R I}-\boldsymbol{\Psi}_{i} \boldsymbol{\mu}_{i}\right\|_{2}^{2}\right),
\end{aligned}
$$

where $\mu_{i, g}^{2}$ and $\mu_{i, g+G}^{2}$ are the $g$ th and $(g+G)$ th elements in vector $\boldsymbol{\mu}_{i}$, and $\Sigma_{i, g g}$ and $\Sigma_{i,(g+G)(g+G)}$ are the $(g, g)$ and $(g+G, g+G)$ entries in matrix $\boldsymbol{\Sigma}_{i}$. Note that, because $\boldsymbol{\alpha}^{(\text {new })}$ and $\beta_{0}^{(\text {new })}$ are a function of $\boldsymbol{\mu}_{i}$ and $\boldsymbol{\Sigma}_{i}$, while $\boldsymbol{\mu}_{i}$ and $\boldsymbol{\Sigma}_{i}$ are a function of $\boldsymbol{\alpha}$ and $\beta_{0}$, this suggests an iterative algorithm that iterates between Eqns. (14)-(15) and Eqns. (21)-(22), until a convergence criterion is satisfied or the maximum number of iterations is reached.

\section{Identifiability}

Since the DOA estimation is separately performed for uncorrelated and coherent targets in two steps, two identifiability conditions are presented below to derive the maximum number of DOFs.

Condition 1: To correctly estimate the DOAs of the uncorrelated targets, the number of dominant eigenvectors in $\mathbf{U}_{s}, D+V$, must satisfy the requirement of $D+V<2 K+1$, or equivalently

$$
D+V \leq 2 K=M N-1 \text {. }
$$

Condition 2: To correctly estimate the DOAs of the coherent targets, the total number of coherent targets $L=\sum_{v=1}^{V} L_{v}$ cannot exceed a half of the number of sum coarray sensors, $K+1$, i.e.,

$$
L \leq K=\left\lfloor\frac{M N-1}{2}\right\rfloor
$$


Proof: In sparse reconstruction, the spark of $\tilde{\mathbf{A}}$, i.e., $\operatorname{spark}(\tilde{\mathbf{A}})$, decides when such recovery is possible. Therefore, following the results in $[33,34]$, the $L$-sparse vector can be uniquely recovered if and only if

$$
L<\frac{\operatorname{spark}(\tilde{\mathbf{A}})-1+\operatorname{rank}(\mathbf{z})}{2}=\frac{2 K+1+\operatorname{rank}(\mathbf{z})}{2}=K+1,
$$

where $\operatorname{spark}(\tilde{\mathbf{A}})=2 K+2$ and $\operatorname{rank}(\mathbf{z})=1$ with $\mathbf{z}=\left[\mathbf{z}_{-K}, \ldots, \mathbf{z}_{K}\right]$. That is, $L \leq K=\left\lfloor\frac{M N-1}{2}\right\rfloor$.

Proposition 1: When Conditions 1 and 2 hold, the maximum number of targets that can be resolved by the proposed approach, $Q=D+L$, is $\lfloor(3 M N-5) / 2\rfloor$.

Proof: Based on the Condition 1, we know that the maximum number of resolvable uncorrelated targets is achieved when the group number is $V=1$. Thus, we can estimate a maximum number of $D=M N-2$ uncorrelated targets plus $\lfloor(M N-1) / 2\rfloor$ coherent targets (Condition $2)$, yielding a total number of resolvable targets to be $\lfloor(3 M N-5) / 2\rfloor$.

From Proposition 1, it is noted that we can resolve a much higher number of targets than the number of physical sensors, $M+N$, and the number of sum coarray elements, $M N$, when $M N \geq 6$.

\section{Simulation Results}

For illustrative purpose, we consider a MIMO radar with a generalized coprime pair of ULAs, where $M=4$ and $N=3$ are assumed and different values of the compression factor $p=1$ and $p=4$ are exploited. As such, all configurations consist of $M+N=7$ physical antennas, yielding $M N=12$ elements in the sum coarray. The number of resolvable targets is upper bounded by $\lfloor(3 M N-5) / 2\rfloor=15$.

Assume that $Q$ mixed uncorrelated and coherent far-field targets are received at the sum coarray, in which $L=\sum_{v=1}^{V} L_{v}$ coherent targets are grouped into $V$ groups in addition to $D$ uncorrelated targets. The covariance matrix are obtained by using 500 snapshots. The grid interval of the spatial angle used in both MUSIC and CS-based approaches is $0.1^{\circ}$. The DOA estimation performance is examined in terms of resolution and accuracy. Particularly, the proposed method is compared with the MUSIC with spatial smoothing (MUSIC-SS) [4] and the approach in [6] (refer to as the $\mathrm{Xu}$ method hereafter).

\section{A. Sum coarray without missing positions}

We first consider the case where the sum coarray does not have missing positions. Such coarrays can be obtained from a nested array structure $(p=4)$. The yielding sum coarray is 
shown in Fig. 2(c). In this case, the proposed method and the $\mathrm{Xu}$ method have the same DOFs to resolve up to $\lfloor(3 M N-5) / 2\rfloor=15$ targets, whereas the MUSIC-SS method can only resolve $(M N / 2-1)=5$ targets. The main difference between the proposed method and the Xu method lies in the utilization of the CS method which offers higher resolution capability as compared to the MUSIC counterpart [35].

In the first example, we consider $V=2$ groups of $Q=L=4$ coherent targets which arrive from $\left[42^{\circ},-40^{\circ}\right]$ and $\left[-12^{\circ},-8^{\circ}\right]$ with coefficients $\boldsymbol{\rho}_{1}=[-0.0349+j 0.9994,-0.6490+$ $j 0.2622]^{T}$ and $\boldsymbol{\rho}_{2}=[0.7092+j 0.5541,0.7999+j 0.0140]^{T}$. The input signal-to-noise ratio (SNR) of all impinging target signals is assumed to be $0 \mathrm{~dB}$. The obtained spectra for the three methods are compared in Fig. 3. It is evident that only the proposed method correctly identify the two closely spaced targets.

Next, we compare the average root mean-square error (RMSE) performance of the estimated DOAs obtained from 200 Monte Carlo trials, expressed as

$$
\mathrm{RMSE}=\sqrt{\frac{1}{200 Q} \sum_{n=1}^{200} \sum_{q=1}^{Q}\left(\hat{\theta}_{q}(n)-\theta_{q}\right)^{2}},
$$

where $\hat{\theta}_{q}(n)$ is the estimate of $\theta_{q}$ for the $n$th Monte Carlo trial, $n=1, \ldots, 200$.

To enable comparison of the RMSE performance among all the three methods, we consider the same $Q=L=4$ target scenario, but the DOAs of targets are changed to $\left[42^{\circ},-40^{\circ}\right]$ and $\left[-12^{\circ}, 9^{\circ}\right]$, i.e., the two targets in the second pair are now widely separated. Fig. 4 shows the RMSE results with respect to the input SNR. It is clear that the proposed method outperforms $\mathrm{Xu}$ method when the input $\mathrm{SNR}$ is higher than $-7 \mathrm{~dB}$, and is approximately the same as the MUSIC-SS when the input SNR is above $-2 \mathrm{~dB}$. On the other hand, the proposed method yields performance degradation when the input SNR is low, as CS-based technique are known to suffer when the input signals are highly noisy.

In the next example, we add $D=9$ uncorrelated targets to the current scenario with $L=4$ coherent targets. The uncorrelated atrgets are uniformly distributed between $\left[-60^{\circ}, 60^{\circ}\right]$. In this case, the total number of targets becomes $Q=D+L=13$. Because the MUSIC-SS fails to function as $Q$ is larger than the number of its resolvable targets, we only compare the results of the proposed method and the $\mathrm{Xu}$ method. Fig. 5 shows similar results that the proposed method yields a lower RMSE when the input SNR is higher than $-6 \mathrm{~dB}$. 


\section{B. Sum coarray with missing positions}

Coprime arrays generally yield missing coarray positions in the sum coarray. In this case, the proposed method can utilize all the coarray sensors whereas the Xu method can only exploit the contiguous coarray sensor positions. As such, it is expected that the proposed method provide higher number of DOFs and enhanced DOA estimation performance.

As an example, we consider the coprime array with $p=1$. The resulting sum coarray is shown in Fig. 2(a), which contains 6 contiguous coarray sensors and 6 discontiguous ones. As such, the maximum number of coherent targets the proposed method can resolve is $L=5$. For the $\mathrm{Xu}$ method, we can obtain a rank-3 Toeplitz matrix, resulting in a maximum number of 2 resolvable coherent targets. The performances of the proposed method and the Xu method are compared under the same scenario of the targets as considered in Fig. 5, where $D=9$ uncorrelated and $L=4$ coherent targets are present.

For uncorrelated targets, both the proposed method and the Xu method use the MUSIC to estimate the DOAs, and the corresponding MUSIC spectra are shown in Fig. 6(a). It is clear that all $Q=9$ uncorrelated targets are correctly identified. On the other hand, the DOA estimation results of the coherent targets are compared in Fig. 6(b). It is clear that only the proposed method resolves all $L=4$ coherent targets, whereas the Xu method fails to provide their correct DOA estimates because, as we discussed above, the number of coherent targets exceeds the maximum number that the $\mathrm{Xu}$ method can resolve.

To compare the RMSE performance between the proposed method and the Xu method for the sum coarray with missing positions, we choose $L=2$ coherent targets with DOAs $\left[42^{\circ},-40^{\circ}\right]$ so that both methods can resolve the targets. The results are compared in Fig. 7 with respect to the input SNR. It is evident that, because of the higher number of DOFs utilized in the proposed method, it achieves significant improvement over the Xu method for all the input SNR values being evaluated.

\section{Comparison between sum coarrays with and without missing positions}

The last example is designed to compare the performance between different coprime MIMO structures that respectively yield sum coarrays with and without missing coarray sensor positions. We used the same MIMO array sensors with $M=4$ and $N=3$ as discussed in the previous examples, where different values of interelement spacing compression factors, i.e., $p=1, p=2$, and $p=4$, are considered. As we can verify from Figs. 2(a)-2(b), all the three sum coarrays have 
12 coarray sensors, but the $p=1$ case has a largest coarray aperture, whereas the $p=4$ case has uniform linear sum coarray. As such, when the proposed method is used, it can benefit from the extended aperture for a small of value of $p$ when estimating the DOAs of both uncorrelated and coherent targets. On the other hand, the existence of missing coarray sensor positions may result in high sidelobes and spurious spectrum estimates.

Assume that $D=2$ uncorrelated targets are located at $\left[0^{\circ}, 3^{\circ}\right.$, and $L=4$ coherent targets are located at $\left[42^{\circ},-40^{\circ}\right]$ and $\left[-12^{\circ},-10^{\circ}\right]$, respectively. In addition, the input SNR of all targets is set to $-3 \mathrm{~dB}$. Fig. 8(a) compares the MUSIC spectra of the uncorrelated targets, where it is clear that only the case of $p=1$ can resolve the closely spaced uncorrelated targets. For the DOA estimation of the coherent targets as shown in Fig. 8(b), the $p=4$ case again fails to resolve the two closely spaced targets at $-12^{\circ}$ and $-10^{\circ}$, whereas the $p=1$ case shows spurious target at $-44^{\circ}$. In this specific example, the $p=2$ case resolves all the targets without spurious estimates.

\section{CONCLUSions}

We have examined the direction-of-arrival (DOA) estimation problem of both uncorrelated and coherent targets in a multiple-input multiple-output (MIMO) platform by using a coprime pair of uniform linear arrays, which yield a symmetric, but not necessarily uniform linear, sum coarray. After applying the subspace-based approaches for uncorrelated targets, the DOA estimation for coherent targets is achieved using the compressive sensing framework. In particular, the complex multitask Bayesian compressive sensing approach is exploited due to its superior performance. The use of compressive sensing methods eliminates the spatial smoothing procedure for signal decorrelation and, subsequently, the requirement for uniform linear sum coarrays. Simulation results verified that the proposed technique achieves superior performance with a flexible array configuration.

\section{ACKNOWLEDGEMENT}

This work was supported in part by the Office of Naval Research (ONR) under grant N0001413-1-0061.

\section{REFERENCES}

[1] S. Qin, Y. D. Zhang, and M. G. Amin, "DOA estimation of mixed coherent and uncorrelated signals exploiting a nested MIMO system," in Proc. IEEE Benjamin Franklin Symposium on Microwave and Antenna Sub-systems, Philadelphia, PA, Sept. 2014. 
[2] R. O. Schmidt, "Multiple emitter location and signal parameter estimation," IEEE Trans. Antennas Propag., vol. 34, no. 3, pp. 276-280, 1986.

[3] R. Roy and T. Kailath, "ESPRIT - Estimation of signal parameters via rotation invariance techniques," IEEE Trans. Acoust. Speech Signal Process., vol. 17, no. 7, pp. 984-995, 1989.

[4] T.-J. Shan, M. Wax, and T. Kailath, "On spatial smoothing for direction-of-arrival estimation of coherent signals," IEEE Trans. Acoust. Speech Signal Process., vol. 33, no. 4, pp. 806-811, 1985.

[5] S. U. Pillai and B. H. Kwon, "Forward/bachward spatial smoothing techniques for coherent signal identification," IEEE Trans. Acoust. Speech Signal Process., vol. 37, no. 1, pp. 8-15, 1989.

[6] X. Xu, Y. Zhang, and C. Chang, "A deflation approach to direction of arrival estimation for symmetric uniform linear array," IEEE Antennas Wirel. Propag. Lett., vol. 5, no. 1, pp. 486-489, 2006.

[7] R. T. Behrens and L. L. Scharf, "Signal processing applications of oblique projection operators," IEEE Trans. Signal Process., vol. 42, no. 6, pp. 1413-1424, 1994.

[8] M. L. McCloud and L. L. Scharf, "A new subspace identification algorithm signal for high resolution DOA estimation,” IEEE Trans. Antennas Propag., vol. 50, no. 10, pp. 1382-1390, 2002.

[9] R. T. Hoctor and S. A. Kassam, "The unifying role of the coarray in aperture synthesis for coherent and incoherent imaging," Proc. IEEE, vol. 78, no. 4, pp. 735-752, 1990.

[10] E. Fishler, A. Haimovich, R. Blum, D. Chizhik, L. Cimini and R. Haimovich, "MIMO radar: an idea whose time has come," in Proc. IEEE Radar Conf., Philadelphia, PA, Apr. 2004, pp. 71-78.

[11] J. Li and P. Stoica, Eds, MIMO Radar Signal Processing, New York, NY: Wiley, 2009.

[12] P. P. Vaidyanathan and P. Pal, "Sparse sensing with co-prime samplers and arrays," IEEE Trans. Signal Process., vol. 59, no. 2, pp. 573-586, 2011.

[13] S. Qin, Y. D. Zhang, and M. G. Amin, "Generalized coprime array configurations for direction-of-arrival estimation,” IEEE Trans. Signal Process., vol. 63, no. 6, pp. 1377-1390, 2015.

[14] P. Pal and P. P. Vaidyanathan, "Nested arrays: A novel approach to array processing with enhanced degrees of freedom,” IEEE Trans. Signal Process., vol. 58, no. 8, pp. 4167-4181, 2010.

[15] D. L. Donoho, “Compressed sensing,” IEEE Trans. Inf. Theory, vol. 52, no. 4, pp. 1289-1306, 2006.

[16] R. Tibshirani, "Regression shrinkage and selection via the lasso," J. R. Stat. Soc., Ser. B, vol. 58, no. 1, pp. 267-288, 1996.

[17] S. S. Chen, D. L. Donoho, and M. A. Saunders, "Atomic decomposition by basis pursuit," SIAM J. Sci. Comput., vol. 20, no. 1, pp. 33-61, 1998.

[18] J. A. Tropp and A. C. Gilbert, "Signal recovery from random measurements via orthogonal matching pursuit," IEEE Trans. Inf. Theory, vol. 53, no. 12, pp. 4655-4666, 2007.

[19] M. E. Tipping, "Sparse Bayesian learning and the relevance vector machine," J. Mach. Learn. Res., vol. 1, no. 9, pp. 211-244, 2001.

[20] S. Ji, Y. Xue, and L. Carin, "Bayesian compressive sensing,” IEEE Trans. Signal Process., vol. 56, no. 6, pp. 2346-2356, 2008. 
[21] S. Ji, D. Dunson, and L. Carin, "Multitask compressive sensing," IEEE Trans. Signal Process., vol. 57, no. 1, pp. 92-106, 2009.

[22] Z. Zhang and D. B. Rao, "Sparse signal recovery with temporally correlated source vectors using sparse Bayyesian learning," IEEE J. Sel. Topics Signal Process., vol. 57, no. 1, pp. 92-106, 2009.

[23] Z. Zhang and D. B. Rao, "Extension of SBL algorithms for the recovery of block sparse signals with intrablock correlation," IEEE Trans. Signal Process., vol. 61, no. 8, pp. 2009-2015, 2013.

[24] Q. Wu, Y. D. Zhang, and M. G. Amin, "Complex multitask Bayesian compressive sensing," in Proc. IEEE Int. Conf. Acoust. Speech Signal Process., Florence, Italy, May 2014, pp. 3375-3379.

[25] S. Qin, Y. D. Zhang, Q. Wu, and M. G. Amin, "DOA Estimation of non-Parametric spreading spatial spectrum based on Bayesian compressive sensing exploiting intra-Task dependency," in Proc. IEEE Int. Conf. Acoust. Speech Signal Process., Brisbane, Australia, April 2015, pp. 2399-2403.

[26] Q. Wu, Y. D. Zhang, M. G. Amin, and B. Himed, "High-resolution passive SAR imaging exploiting structured Bayesian compressive sensing," IEEE J. Sel. Topics Signal Process., vol. 9, no. 8, pp. 1484-1497, 2015.

[27] S. Qin, Y. D. Zhang, and M. G. Amin, "Structure-aware Bayesian compressive sensing for near-field source localization based on sensor-angle distributions," Int. J. Antennas Propagat., vol. 2015, Article ID 783467, 15 pages, 2015.

[28] Q. Wu, Y. D. Zhang, and M. G. Amin, "Continuous structure based Bayesian compressive sensing for sparse reconstruction of time-frequency distributions," in Proc. IEEE Conf. Digital Signal Process., Hong Kong, China, Aug. 2014, pp. 831-836.

[29] F. Liu, J. Wang, C. Sun, and R. Du, "Spatial differencing method for DOA estimation under the coexistence of both uncorrelated and coherent signals," IEEE Trans. Antennas Propag., vol. 60, no. 4, pp. 2052-2062, 2012.

[30] J. Huang and T. Zhang, "The benefit of group sparsity,” Ann. Statist., vol. 38, no. 4, pp. 1978-2004, 2010.

[31] M. Yuan and Y. Lin, "Model selection and estimation in regression with grouped variables," J. R. Stat. Soc., Ser. B, vol. 68, no. 1, pp. 49-67, 2006.

[32] W. R. Gilks, S. Richardson, and D. J. Spiegelhalter, Markov Chain Monte Carlo in Practice, Chapman \& Hall, 1996.

[33] J. Chen and X. Huo, “Theoretical results on sparse representation of multiple-measurement vectors," IEEE Trans. Signal Process., vol. 54, no. 12, pp. 4634-4643, 2006.

[34] M. E. Davis and Y. C. Eldar, "Rank awareness in joint sparse recovery," IEEE Trans. Signal Process., vol. 58, no. 2, pp. 1135-1146, 2012.

[35] M. Carlin, P. Rocca, G. Oliveri, F. Viani, and A. Massa, "Directions-of-arrival estimation through Bayesian compressive sensing strategies," IEEE Trans. Antennas Propagat., vol. 61, no. 7, pp. 3828-3838, July 2013. 


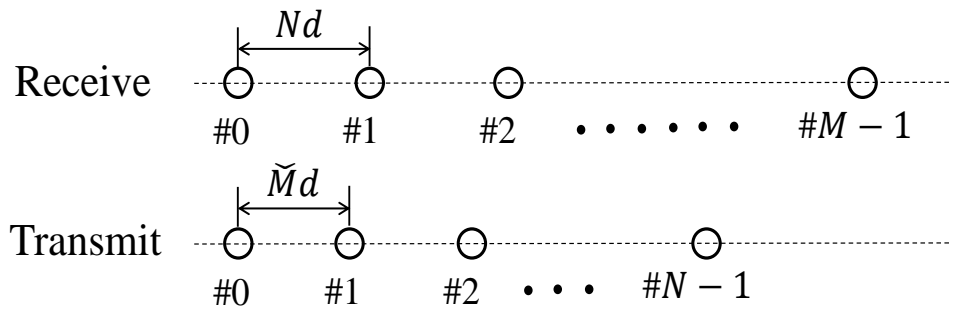

Fig. 1. A MIMO radar with a coprime pair ULA.

$$
\begin{array}{cccccccccccccccccc}
\bullet & \times & \times & \bullet & \bullet & \times & \bullet & \bullet & \bullet & \bullet & \bullet & \bullet & \times & \bullet & \bullet & \times & \times & \bullet \\
0 & 1 & 2 & 3 & 4 & 5 & 6 & 7 & 8 & 9 & 10 & 11 & 12 & 13 & 14 & 15 & 16 & 17
\end{array}
$$

(a) $p=1$

$\begin{array}{cccccccccccccccccc}\bullet & \times & \bullet & \bullet & \bullet & \bullet & \bullet & \bullet & \bullet & \bullet & \bullet & \bullet & \times & \bullet & \times & \times & \times & \times \\ 0 & 1 & 2 & 3 & 4 & 5 & 6 & 7 & 8 & 9 & 10 & 11 & 12 & 13 & 14 & 15 & 16 & 17\end{array}$

(b) $p=2$

$\begin{array}{cccccccccccccccccc}\bullet & \bullet & \bullet & \bullet & \bullet & \bullet & \bullet & \bullet & \bullet & \bullet & \bullet & \bullet & \times & \times & \times & \times & \times & \times \\ 0 & 1 & 2 & 3 & 4 & 5 & 6 & 7 & 8 & 9 & 10 & 11 & 12 & 13 & 14 & 15 & 16 & 17 \\ & \end{array}$

Fig. 2. The coarrays of the MIMO radar with a generalized coprime pair of ULA for different values of compression factor $p$ $(M=4$ and $N=3)$. 


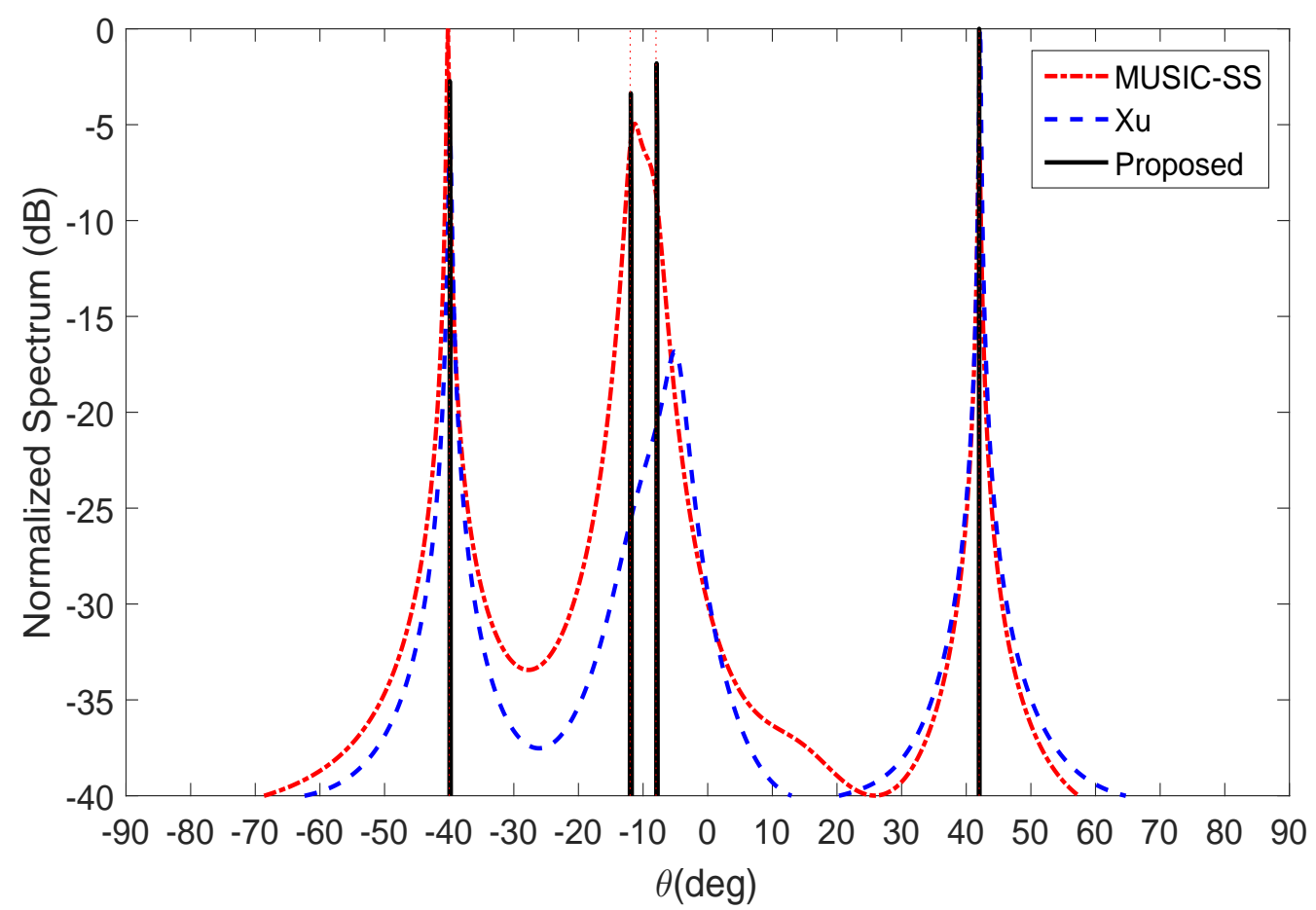

Fig. 3. Estimated spatial spectrum using the MUSIC-SS, Xu and proposed techniques $(p=4$ and $Q=L=4)$.

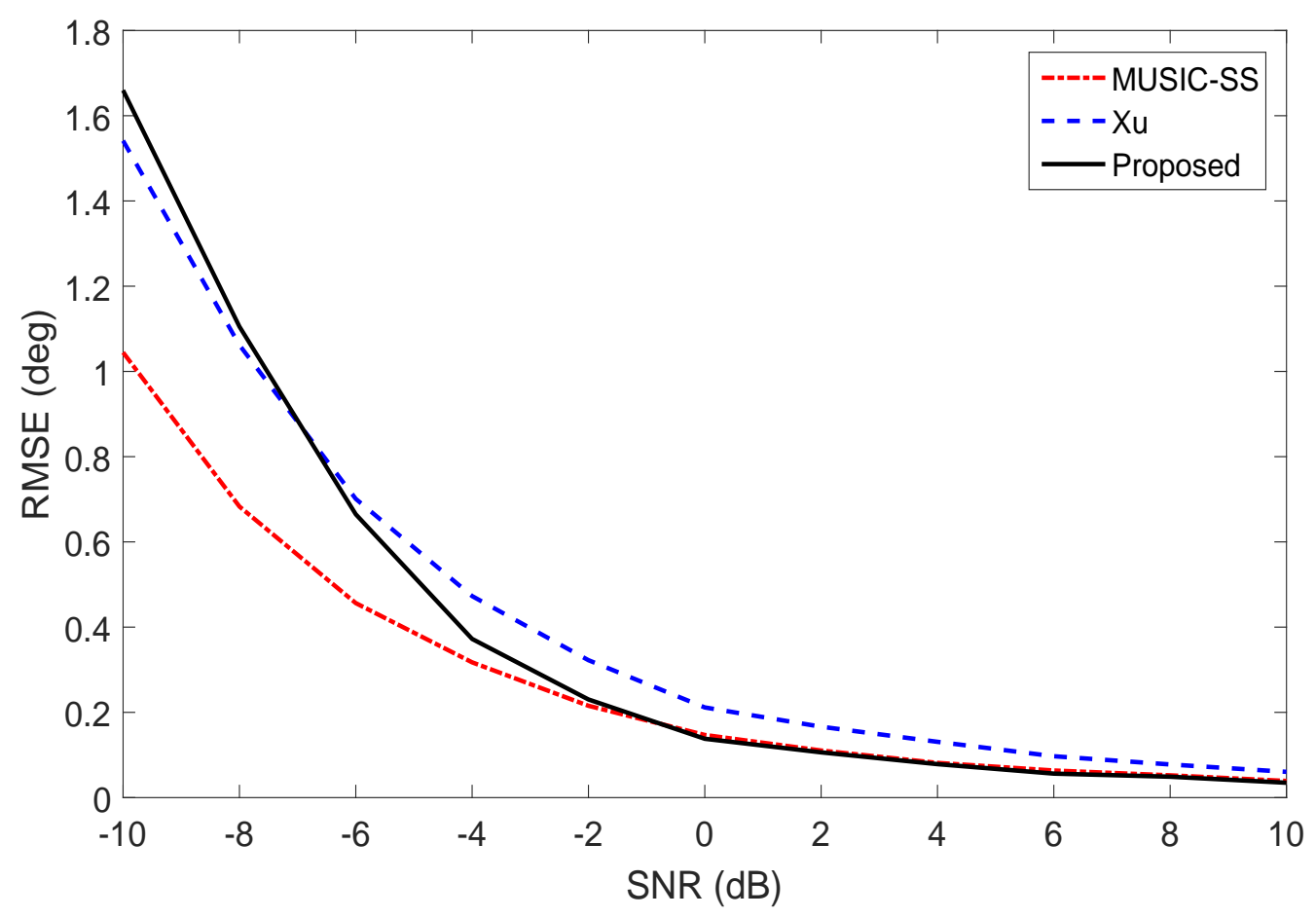

Fig. 4. RMSE versus SNR using the MUSIC-SS, Xu and proposed techniques ( $p=4$ and $Q=L=4$ ). 


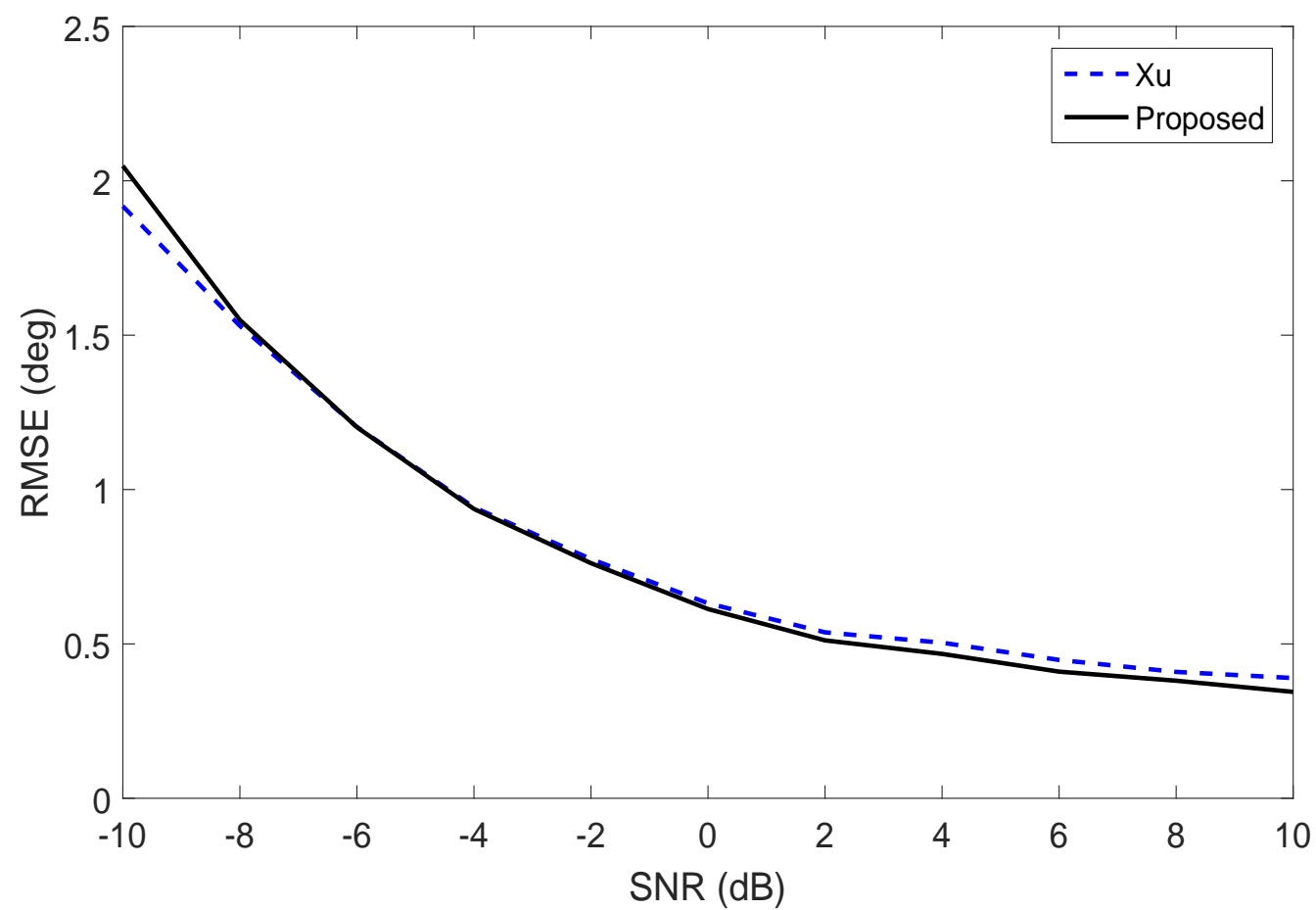

Fig. 5. RMSE versus SNR using the Xu and proposed techniques $(p=4, Q=13, D=9$, and $L=4)$. 


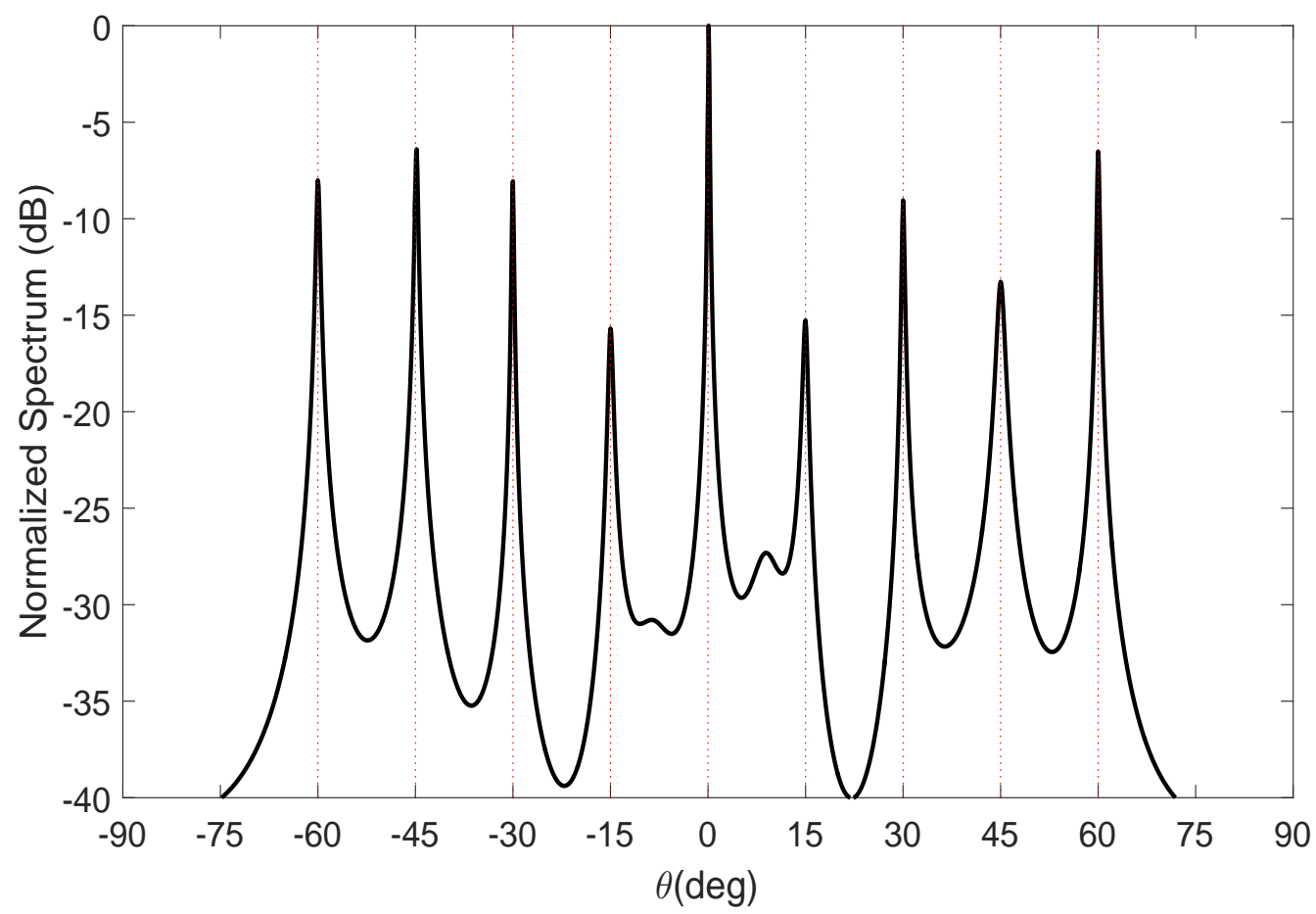

(a)

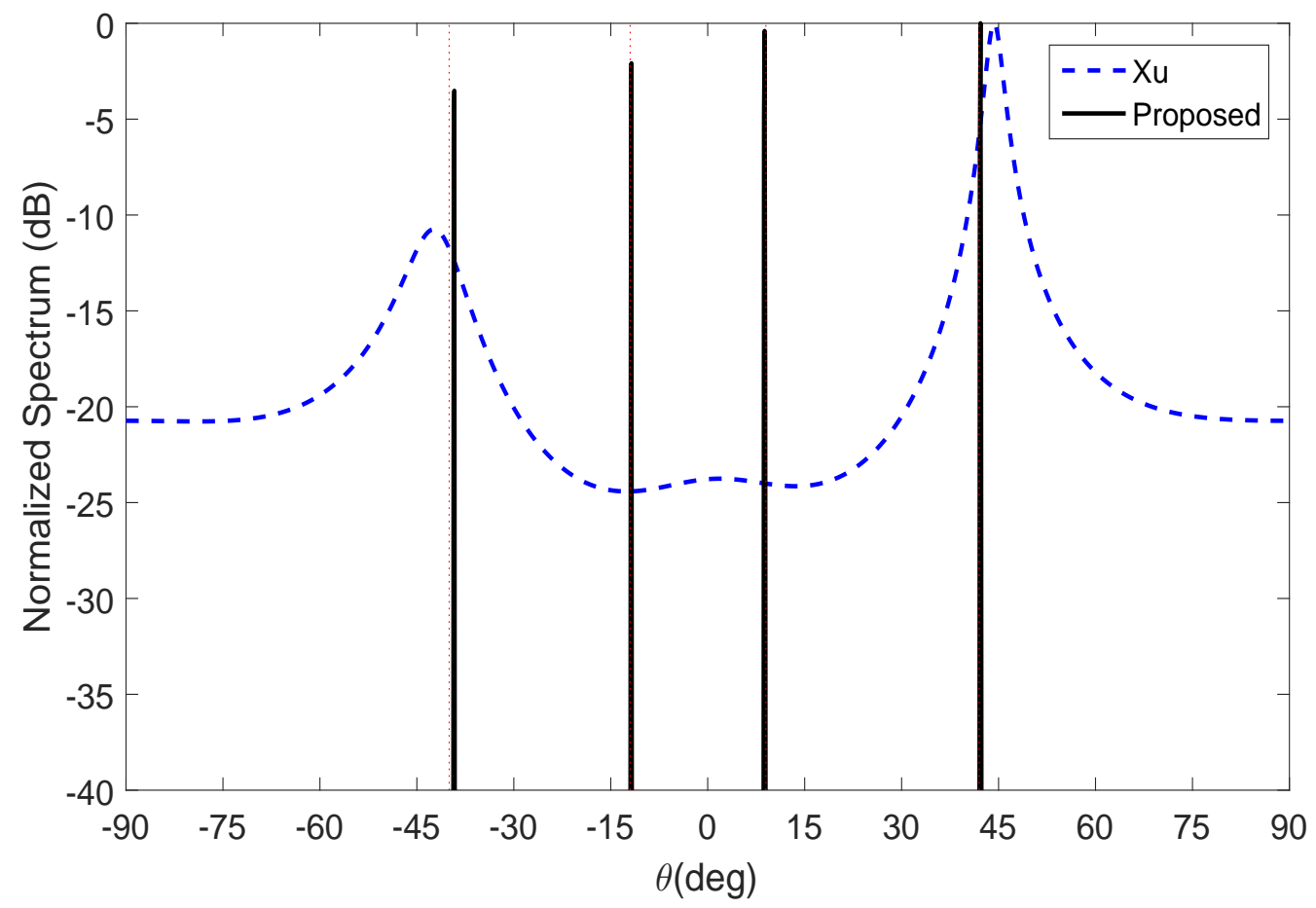

(b)

Fig. 6. Estimated spatial spectrum using the $\mathrm{Xu}$ and proposed techniques ( $p=1, Q=13, D=9$ and $L=4$ ). (a) Uncorrelated signals; (b) Coherent signals. 


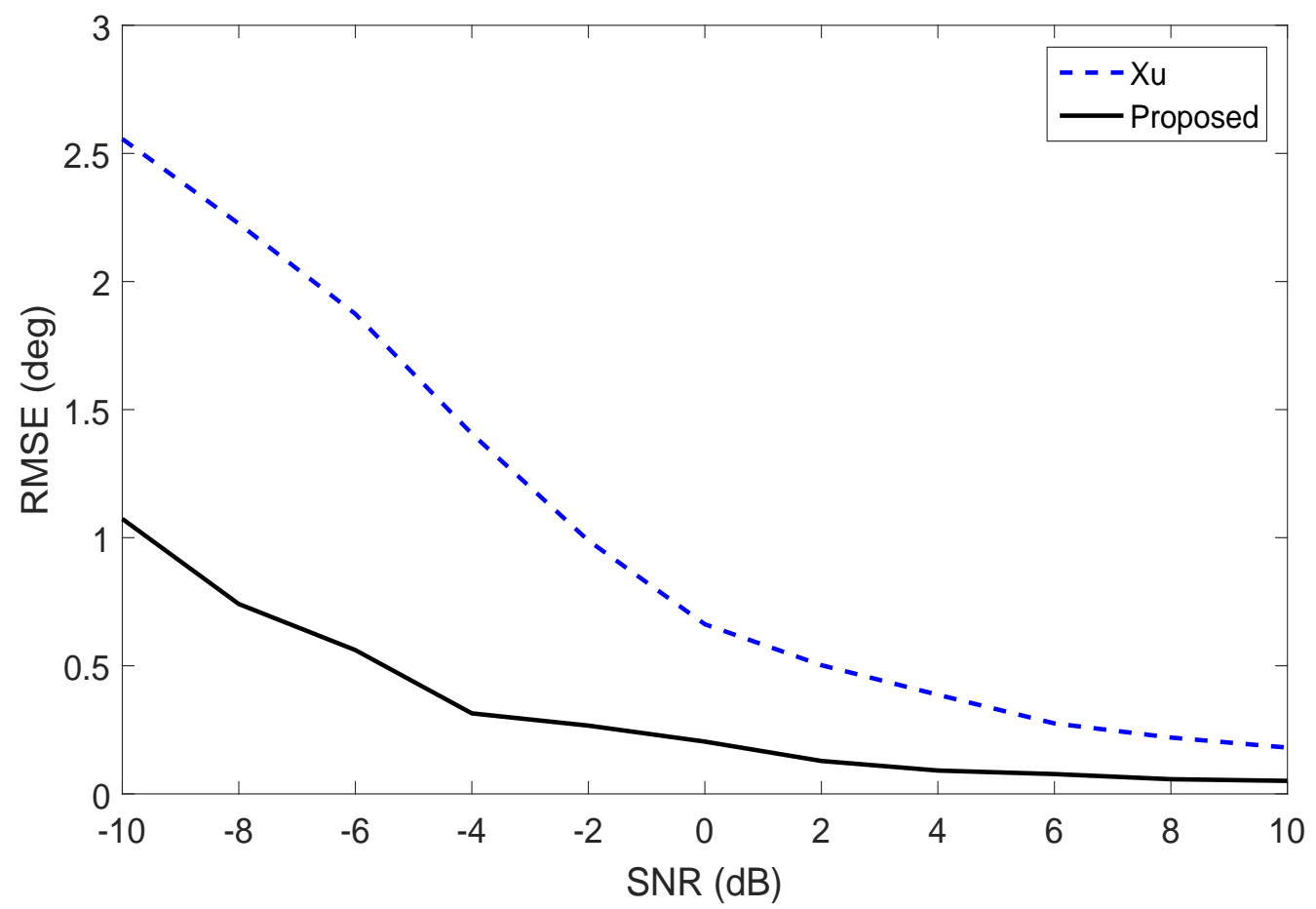

Fig. 7. RMSE versus SNR using the Xu and proposed techniques $(p=1$ and $Q=L=2$ ). 


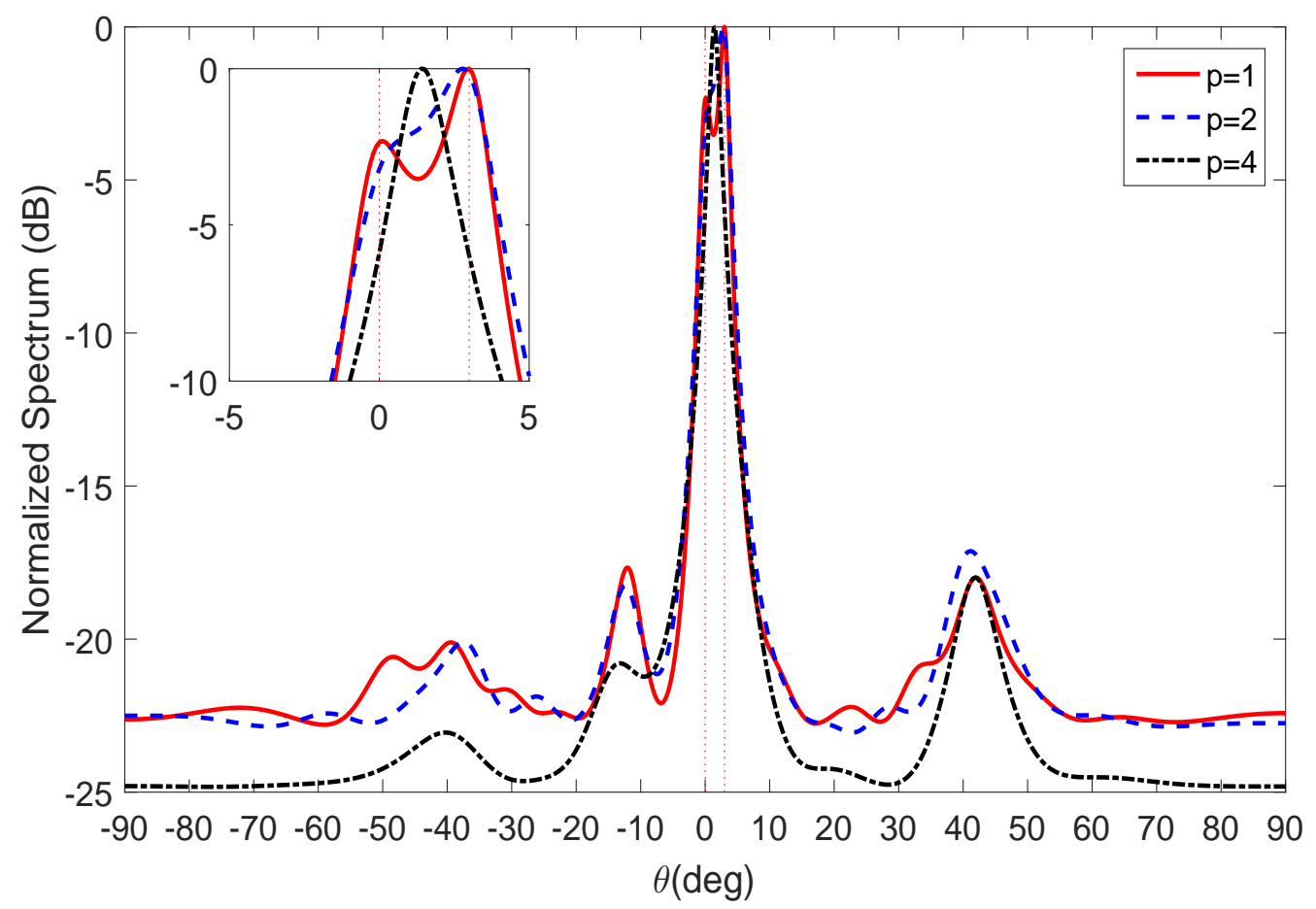

(a)
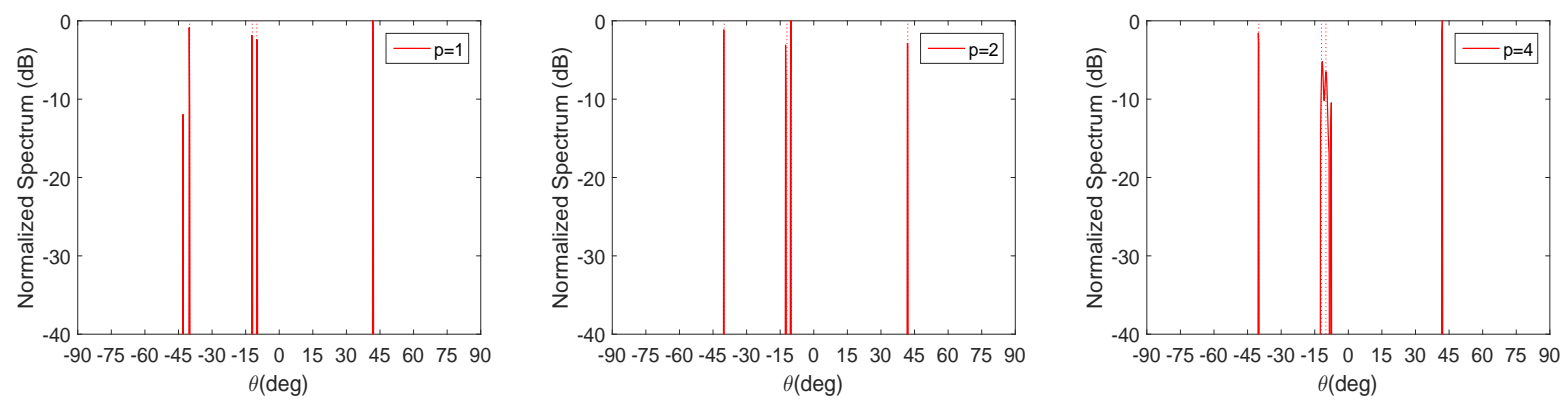

(b)

Fig. 8. Estimated spatial spectrum for the cases of $p=1, p=2$, and $p=4(Q=6, D=2$ and $L=4)$. (a) Uncorrelated signals; (b) Coherent signals. 